\title{
On the Application of the Simplified Crack Model in the Bending, Free Vibration and Buckling Analysis of Beams with Linear Variation of Widths
}

\author{
Matjaž Skrinar ${ }^{1 *}$ \\ ' Department of Structural Mechanics, \\ Faculty of Civil Engineering, Transportation Engineering and Architecture, \\ University of Maribor, Smetanova 17, 2000 Maribor, Slovenia \\ *Corresponding author, e-mail: matjaz.skrinar@um.si
}

Received: 15 June 2018, Accepted: 28 January 2019, Published online: 25 March 2019

\begin{abstract}
Adequate computational models are crucial for a reliable representation of the mechanical behaviour of structural elements and, therefore, numerous investigations are oriented towards the modelling of cracked structures.

This paper studies the behaviour of transversely cracked beams of rectangular cross-sections with linearly-varying widths. The governing differential equations of bending are analytically solved for various beams with different boundary conditions. These simplified model's solutions are further validated by the results from corresponding 3D finite models of the considered structures. Furthermore, strain and kinetic energy, as well as the work done by an external axial compressive force P are evaluated from the computed transverse displacement functions. These values allowed for estimations of the first eigenfrequency as well as the buckling load. These structural's parameters were additionally evaluated by implementing dedicated polynomial functions for some cases considered. The results from the simplified model have exhibited very good agreement with the results from more detailed 3D FE models for all performed analyses. The simplified model thus yields an adequate, as well as accurate, approach for the modelling of cracked beams with a linear variation of width in engineering situations, where cracks have to be considered during analysis.
\end{abstract}

\section{Keywords}

cracked beams with transverse cracks, linear cross-sections' variations, simplified computational model, governing differential equations of bending, transverse displacements

\section{Introduction}

The occurrence of degenerative effects in structures during the utilisation changes their mechanical response by severely decreasing the stiffness and potentially leading to their failure. In view of this, several studies considering the detection and identification of stiffness reductions in engineering structures were conducted. The approaches for structural damages detection and characterization are often based on measured response methods since the occurrence of damage alters the structures' response data. However, the efficiency of these approaches does not depend only on the quality of measured information but also on the reliabilities of computational models implemented in modelling of mechanical behaviour.

The genuine approach in fracture mechanics is to model the crack as an elliptical hole with the semi-minor axis tending to zero (Craciun [1]). However, when studying cracked structures' response appropriate meshes of 2D or 3D finite elements that allow for a detailed discretisation of the crack and its surroundings represent a widely used alternative.

Although such thorough meshes offer the finest description of a general structure, as well as of the cracks and their surroundings, simplified models requiring fewer data are usually implemented in structural health monitoring techniques. The "discrete spring" model presented by Okamura et al. [2] is the model that has been implemented in numerous research studies. As a crack in a structural member alters the local compliance, each crack in this mathematical representation is modelled as a massless rotational linear spring of appropriate stiffness. The neighbouring noncracked parts of the beam to the left and to the right of the crack are modelled as elastic elements, connected by the spring for which the linear moment-rotation constitutive 
law is adopted. The first definition for rotational spring stiffness was given by Okamura et al. for a rectangular crosssection. Afterwards, other researchers presented additional definitions [3-8].

Due to its simplicity (the only crack's parameters required are its location and the depth), this simplified computational representation has been intensively applied in vibration analysis of cracked beams (Bakhtiari-Nejad et al. [9]), new various approaches for inverse identification of cracks [10-11], as well as in experimental inverse identifications of a crack (Cao et al. [12]) or a concentrated damage (Greco and Pau [13]).

The research consideration focused towards finite elements solution after the pioneering works by Tharp [14] and Gounaris \& Dimarogonas [15] for a beam element with a single transverse crack. Afterwards, several papers were devoted to Euler-Bernoulli beam's finite element having an arbitrary number of transverse cracks differing in the principles of mechanics applied to obtain closed-form solutions of the genuine governing differential equation for transverse displacements. The static transverse displacements and various forms of stiffness matrix were thus obtained by implementing the Dirac delta function either in regard to the rigidity (Biondi and Caddemi [16]) or flexibility (Palmeri and Cicirello [17]), sequential solutions of coupled differential equations (Skrinar [18]), as well as the virtual work principle (Skrinar and Pliberšek [19]).

The majority of the research has been limited to elements with constant rectangular cross-sections. Skrinar and Imamović [20] presented a numerical study in which they studied bending of beams of various heights' variations along the length. In their investigation, they implemented a multistepped multi-cracked beam finite element (Skrinar [21]) where the genuine continuous variation of height was modelled by an adequate series of steps. This model offers adequate, but approximate solutions for bending analyses only.

In this paper, the area of utilization of the simplified crack model is expanded by deriving at some closed-form solutions for bending of beams with linearly-varying widths. Furthermore, additionally derived at model's polynomial displacement functions are implemented in the dynamic and buckling analysis.

\section{Solutions of governing differential equations of bending 2.1 General solution}

The general governing differential equation of the elastic line for a slender beam subjected to bending in the plane of symmetry that relates transverse displacement $v(x)$, the coordinate $x$, the geometrical and mechanical properties of the cross-section (flexural rigidity $E I(x)$ ), and the applied transverse load $q(x)$, known also as Euler-Bernoulli equation is given as:

$$
\frac{d^{2}}{d x^{2}}\left(E I(x) \cdot \frac{d^{2} v(x)}{d x^{2}}\right)=q(x) \quad 0 \leq x \leq L .
$$

If the flexural rigidity $E I(x)$ is not a constant value this relation yields a fourth-order ordinary differential-equation with non-constant coefficients.

When studying the beams with a linear variation of width the flexural rigidity also follows a linear distribution. The variation of flexural rigidity $E I(x)$ within the beam is thus described as:

$E I(x)=E I_{o}+k \cdot x$

with

$k=\frac{E I_{L}-E I_{o}}{L}$,

where $E I_{o}$ and $E I_{L}$ represent flexural rigidities at the start and at the end of the beam, respectively. Although Eq. (3) can be introduced into Eq. (2) prior any further derivations this leads to logarithmic terms with negative arguments in the derived solutions causing numerical issues. However, these problems are completely avoided by introducing the coefficient $k$ which further yields more abbreviate forms of the derived at expressions. Therefore, Eq. (1) obtains the form:

$$
\begin{aligned}
& 2 \cdot k \cdot \frac{d^{3} v(x)}{d x^{3}}+E I_{o} \cdot \frac{d^{4} v(x)}{d x^{4}}+ \\
& k \cdot x \cdot \frac{d^{4} v(x)}{d x^{4}}=q(x) \quad 0 \leq x \leq L
\end{aligned}
$$

The general solution of this differential equation is given in the following mathematical form:

$$
\begin{aligned}
& v(x)=C_{2}+x \cdot\left(C_{3}+\frac{C_{1}}{k^{2}}\right)+C_{4} \cdot x^{2} \\
& -C_{1} \cdot \frac{E I(x)}{k^{3}} \cdot \operatorname{Ln}(E I(x))+\text { particular int. }
\end{aligned}
$$

where $C_{1}, C_{2}, C_{3}$ and $C_{4}$ are constants of the integration obtained from boundary conditions, whilst the particular integral depends on the mathematical form of load $q(x)$. It is instructive to note that this solution is not given in a form of simple polynomials as it is the case for the constant flexural rigidity $E I$. 
For a frequent case when a uniform load $q$ is distributed to the whole beam the particular integral obtains the following form:

$$
-\frac{E I_{o}{ }^{2} \cdot q}{2 \cdot k^{3}} \cdot x+\frac{q}{12 \cdot k} \cdot x^{3}+\frac{E I_{o}{ }^{2} \cdot q \cdot E I(x)}{2 \cdot k^{4}} \cdot \operatorname{Ln}(E I(x))
$$

while for a linear distribution of the transverse load the particular integral already becomes significantly more complex.

\subsection{Implementation of continuity conditions}

However, the crack, located arbitrarily within the beam $\left(0 \leq L_{1} \leq L\right)$, separates the beam into two elastic parts, and the transverse displacements cannot be described by a single function anymore. Therefore, two displacement functions for the parts on the left $\left(v_{1}(x)\right)$ and right $\left(v_{2}(x)\right)$ side of the crack are required. These functions are obtained from two coupled differential equations that have to be solved simultaneously. Their solutions - functions $\left(v_{1}(x)\right)$ and $\left(v_{2}(x)\right)$ for the parts to the left and the right, respectively, contain eight unknown constants altogether:

$v_{1}(x)=C_{2}+x \cdot\left(C_{3}+\frac{C_{1}}{k^{2}}\right)+C_{4} \cdot x^{2}$

$-C_{1} \cdot \frac{E I(x)}{k^{3}} \cdot \operatorname{Ln}(E I(x))+$ particular int.

$v_{2}(x)=D_{2}+x \cdot\left(D_{3}+\frac{D_{1}}{k^{2}}\right)+D_{4} \cdot x^{2}$

$-D_{1} \cdot \frac{E I(x)}{k^{3}} \cdot \operatorname{Ln}(E I(x))+$ particular int.

Four of them are determined from mechanical and kinematical continuity conditions at the crack-location $\left(x=L_{1}\right)$, where the presence of the crack causes a slope discontinuity. These conditions are the equality of displacement, the condition for the discrete increase of rotations, the equality of bending moments, and the equality of shear forces.

Since the continuity conditions are the same regardless of the boundary conditions four out of eight unknown constants can be eliminated in advance. The introduction of Eqs. (7) and (8) and their derivatives into the continuity conditions thus allow the coefficients of the second function to be determined. They are clearly presented in Appendix A, where $E I_{1}$ denotes the flexural rigidity of the non-cracked cross-section at the crack location. The discussed approach can be also straightforwardly expanded to cover a situation with an arbitrary number of cracks. A generalisation of this process in which the number of unknowns is decreased by applying the continuity conditions is in references known as Transfer matrix method, (first introduced by Pestel and Leckie [22]).

The remaining four coefficients of the first function are always determined from the actual boundary conditions of each considered example. With the solutions for the displacements of the beam being available, the bending moments and shear forces distributions within the beam can be calculated afterwards. The presented way allows the transverse displacements to be constructed in form of analytical solutions for some beams with simple boundary conditions and typical load situations.

\subsection{Cantilever beam}

The coefficients for a cantilever beam clamped at the right end are presented in Appendix B. They were obtained from two mechanical conditions at the free end and two kinematical conditions at the clamped end.

\subsection{Simply supported beam}

When considering a simply supported beam a kinematical plus a mechanical condition are available at each beam's end and the corresponding coefficients can be found in Appendix C.

\subsection{Propped cantilever}

For the beam, clamped at the left end the corresponding coefficients obtained from a single mechanical and three kinematical boundary conditions are written in a partially mutually related form (see Appendix D).

\subsection{Beam clamped at both ends}

Appendix E represents the coefficients $C_{3}$ in $C_{4}$ for the last type of the considered beams, where all the coefficients are obtained exclusively from kinematical boundary conditions. The expressions for the coefficients $C_{1}$ in $C_{2}$ are identical as for the propped cantilever, Eqs. (D.1) and (D.2).

\section{Eigenfrequency and buckling analyses implementing the energy method}

For the situation where the breathing of the crack is not considered i.e. the crack remains open, an adequate estimation of the first eigenfrequency can be established also by the Rayleigh energy method (also known as the energy method), Koloušek [23]. The "strain" energy (the potential energy stored as elastic deformation of the structure including crack) is approximated as: 


$$
\begin{aligned}
& U_{\text {strain }}=\frac{1}{2} \cdot\left(\int_{x=0}^{L_{1}} E I(x) \cdot\left(v_{1}{ }^{\prime \prime}(x)\right)^{2} \cdot d x+\right. \\
& \left.\int_{x=L_{1}}^{L} E I(x) \cdot\left(v_{2}{ }^{\prime \prime}(x)\right)^{2} \cdot d x+K_{r} \cdot\left(v_{1}{ }^{\prime}\left(L_{1}\right)-v_{2}{ }^{\prime}\left(L_{1}\right)\right)^{2}\right) .
\end{aligned}
$$

In Eq. (9) functions $v_{1}(x)$ and $v_{2}(x)$ are functions that represent the transverse displacements and must satisfy the most important kinematical boundary conditions, such as displacement and rotation. In the absence of an exact solution of the differential equation, approximate functions are applied, where static deflection functions represent a wide group of suitable functions since they automatically satisfy the required kinematic boundary conditions. Despite this, these assumed functions are usually constructed from a dedicated polynomial solution, primarily due to ease of their integration. Among the results obtained from various assumed displacement functions, the smallest value yields an upper limit of the true fundamental frequency. Therefore, the already discussed transverse displacements function due to bending, Eqs. (7) and (8), allow for very straightforward implementation.

To obtain the first eigenfrequency estimation the beam's kinetic energy is approximated as

$$
U_{k i n}=\frac{\omega_{1}^{2}}{2}\left(\int_{x=0}^{L_{1}} m(x) \cdot\left(v_{1}(x)\right)^{2} \cdot d x+\int_{x=L_{1}}^{L} m(x) \cdot\left(v_{2}(x)\right)^{2} \cdot d x\right) .
$$

Afterwards, the first in-plane vibrations eigenfrequency estimate is obtained from the total mechanical energy conservation law:

$U_{\text {strain }}=U_{\text {kin }}$.

The results from these functions $v(x)$ can be improved by evaluating new upgraded displacement functions due to a transverse load, given as $q(x)=m(x) \cdot \omega^{2} \cdot v(x)$.

Additionally, the same transverse displacements functions can be further implemented to evaluate the work (i.e. "applied" energy) done on the system by an external axial compressive force $\mathrm{P}$ :

$U_{\text {app }}=\frac{P}{2}\left(\int_{x=0}^{L_{1}}\left(v_{1}^{\prime}(x)\right)^{2} \cdot d x+\int_{x=L_{1}}^{L}\left(v_{2}^{\prime}(x)\right)^{2} \cdot d x\right)$.

Since the energy is not dissipated as heat the energy conservation law states that energy added to the column by the applied external forces is equal to the stored strain energy, yielding the relation:

$U_{\text {strain }}=U_{\text {app }}$, from which the estimate of the buckling load $P_{c r i t}$ can be evaluated. Strategies for the improvement of initial solutions can be found in [24].

\section{Numerical validations}

Four cracked basic beam-structures were analyzed in order to investigate the presented expressions and to verify the quality of the results. For all four structures that differed only in boundary conditions, the length $L$ was $10 \mathrm{~m}$ and the Young modulus was $30 \mathrm{GPa}$ with Poisson's ratio 0.3. The cross-section was a rectangle with height $h=0.2 \mathrm{~m}$ where the width $b$ was linearly increasing from $0.1 \mathrm{~m}$ at the leftend to $0.2 \mathrm{~m}$ at the right-end. All considered structures were loaded by a downward vertical uniform load $q=2000 \mathrm{~N} / \mathrm{m}$ along the complete structure. A single transverse crack of the depth of $0.1 \mathrm{~m}$ was located at the mid-span to maximise its impact on the results for the majority of the examples, and the definition given by Okamura was selected.

To obtain the first eigenfrequency and the buckling load estimations, basic polynomial functions were constructed considering general boundary conditions only. These functions were further introduced into Eqs. (9-13). Afterwards, these functions were upgraded accordingly to the specific problem.

The obtained results were further compared with the values from a commercial finite element program COSMOS/M where corresponding 3D finite models of the considered structures were established and analyzed. The computational model consisted of 48,000 3D solid finite elements with almost 75,000 nodal points. In each node, three degrees of freedom were taken into account - vertical and two horizontal displacements. The model's vertical and horizontal displacements were obtained in discrete points by solving more than 220,000 linear equations. Since this model allows for a realistic description of the crack those results further served as the reference values.

For all the considered structures the results for bending analyses are presented also for the non-cracked cases. By presenting these results the impact of the crack to the magnitude, as well as, the quality of the results become more apparent.

\subsection{Cantilever, clamped at the right end}

After inserting the corresponding numerical values the following transverse displacements' functions were obtained:

$$
\begin{aligned}
& v_{1}(x)=-98.722-10.800 \cdot x-0.03437 \cdot x^{2} \\
& +1.146 \cdot 10^{-3} \cdot x^{3}+6.873 \cdot \operatorname{Ln}\left(2 \cdot 10^{6}+200000 \cdot x\right) \\
& +0.687 \cdot x \cdot \operatorname{Ln}\left(2 \cdot 10^{6}+200000 \cdot x\right) \quad 0 m \leq x \leq 5 \mathrm{~m},
\end{aligned}
$$




$$
\begin{aligned}
& v_{2}(x)=-98.759-10.792 \cdot x-0.03437 \cdot x^{2} \\
& +1.146 \cdot 10^{-3} \cdot x^{3}+6.873 \cdot \operatorname{Ln}\left(2 \cdot 10^{6}+200000 \cdot x\right) \\
& +0.687 \cdot x \cdot \operatorname{Ln}\left(2 \cdot 10^{6}+200000 \cdot x\right) \quad 5 m \leq x \leq 10 m .
\end{aligned}
$$

The transverse displacement at the free end was $-0.7275 \mathrm{~m}$, whilst the rotation's value was $0.1019 \mathrm{rad}$.

The obtained functions allowed for the computation of bending moments' and shear forces' functions which were identical to those obtained from the basic static equilibrium analysis. Further, discrete vertical reaction force and bending moment at the clamped-end were evaluated. These two reaction values perfectly matched the exact values which can be simply verified by elementary equilibrium conditions.

Finally, the structure was also analysed by implementing the COSMOS/M commercial finite element program. This model produced the free end's vertical displacement of $-0.7272 \mathrm{~m}$. This value confirms excellent result from the simplified model which has produced a value with a rather small discrepancy $(0.032 \%)$. However, it is interesting to note that the discrepancy at the crack location is slightly higher $(0.142 \%)$. Nevertheless, the general matching of the results between the two models is actually very good for all the point along the cantilever, Fig. 1, where continuous displacements' functions (in black) from the GDE's solutions visually completely coincide with the discrete nodal values from the 3D model (in black). The over-all matching of the results between the two models was very good also for the non-cracked case (presented in grey), Fig.1, where the free end's discrepancy was $0.069 \%$.

The example was additionally analysed with different ratios $E I_{0} / E I_{L}$ between 0.1 and 10 , utilising the already presented models where the values for the free end's displacement were scrutinised. It emerged from the results that

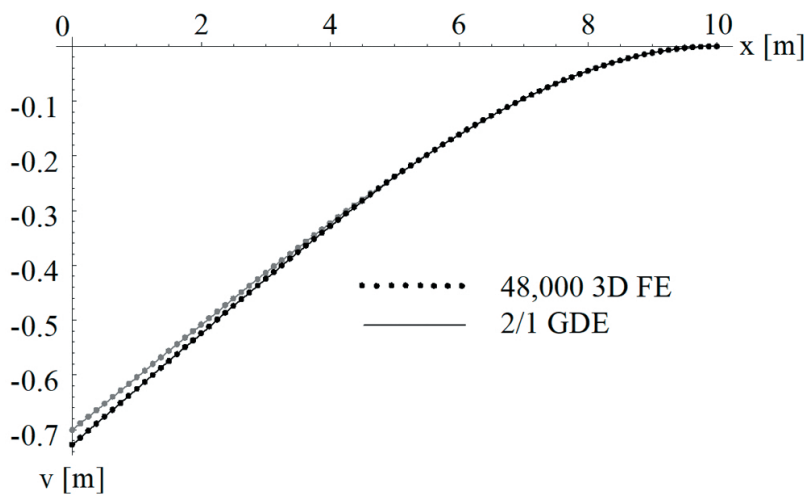

Fig. 1 Comparison of transverse displacements from both applied models the discrepancies between the models increased simultaneously with the increase of cantilever's width i.e. with the transformation of a beam-type structure into a plate-type structure. Furthermore, it became clear that the increase of the width at the clamped side (where it has more impact on structure's stiffness and free end's displacement) influences the discrepancies more than the increase of the width at the free end. Nevertheless, the error was still smaller than $1 \%$ which again confirmed the quality of the results.

To obtain the first eigenfrequency estimation from Eqs. (9-11) polynomial functions were generated by implementing basic general boundary conditions. This resulted in the value $\omega_{1}=8.529 \mathrm{rad} / \mathrm{s}$ for the first eigenfrequency estimation. On the other hand, the 3D FE model produced the value of $8.495 \mathrm{rad} / \mathrm{s}$ again confirming good quality result from the simplified model as the discrepancy between the two models' results is $0.408 \%$.

Further, the buckling load $P_{c r i t}$ was approximated from Eqs. (12) and (13) by implementing the same basic polynomial functions. The buckling load estimation was $81818.4 \mathrm{~N}$. Alternatively, the 3D FE model produced the value of $76724.8 \mathrm{~N}$ showing that the simplified model produced the results with a rather high discrepancy of $6.64 \%$.

Afterwards, the upgrade of basic polynomial functions was performed separately for first eigenfrequency estimation as well as for buckling. The newly derived at upgraded polynomial approximations for the first eigenfrequency estimation produced the value $\omega_{1}=8.493 \mathrm{rad} / \mathrm{s}$, which has a rather low discrepancy $(-0.022 \%)$ against the $3 \mathrm{D}$ model value. However, it should be noted that the obtained value underestimates the value from the $3 \mathrm{D}$ model. This is a consequence of the computational model and not of the method, as the approximate method is being applied to a simplified model.

The upgrade of initial polynomial functions was executed also for the buckling problem. A new set of transverse displacements functions were derived at by realising that in buckling the transverse displacements are a sole function of axial compressive force $P_{c r i t}$. Therefore, the bending moments' functions were expressed as functions of applied axial force and transverse displacements. The considered problem's specific relation was $M_{z}(x)=P_{c r i t} \times$ $(v(0)-v(x)$. With these two new functions for the rotations the "strain" energy, Eq. (9), as well as the "applied" energy, Eq. (12), were re-evaluated. Finally, Eq. (13) yielded the improved value for the buckling load of $P_{c r i t}=76856.7 \mathrm{~N}$ with a small discrepancy of $0.172 \%$. 


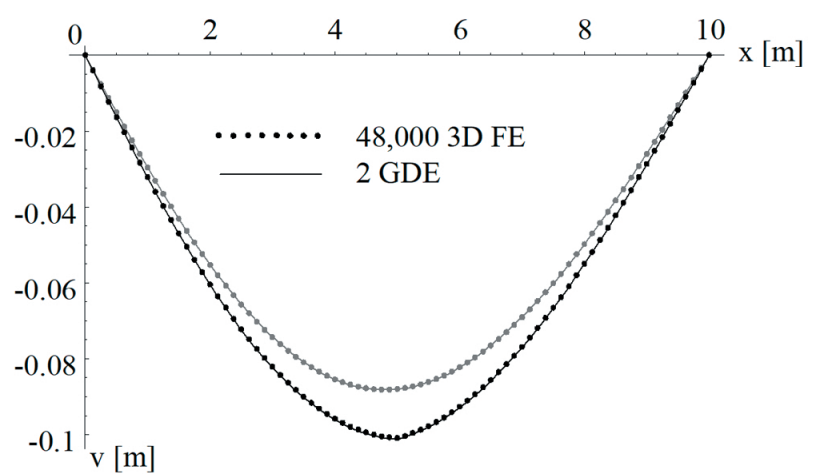

Fig. 2 Comparison of transverse displacements from both applied models

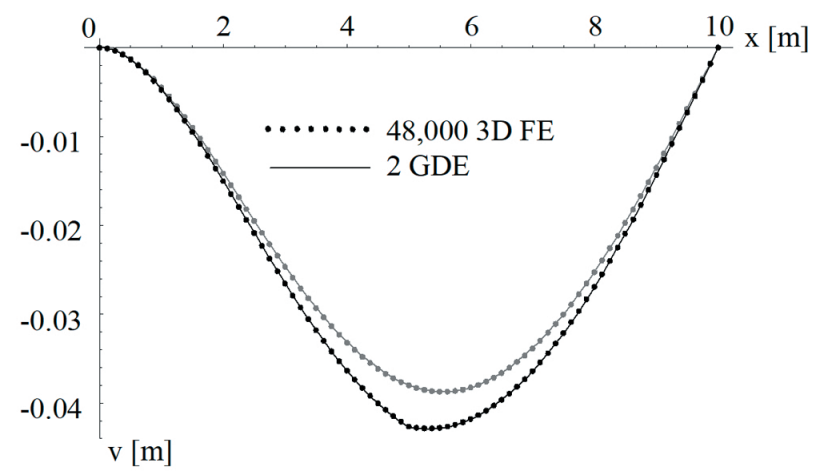

Fig. 3 Comparison of transverse displacements from both applied models

\subsection{Simply supported beam}

Comparison of transverse displacements along the whole structure from GDE's solutions (continuous function), as well as from 3D FE model (discrete nodal values) is given in Fig. 2 (in black). It emerges from the figure that the results from both models appear almost as a unique solution. A similar situation is evident also for the non-cracked case (presented in grey).

Some representative example's values are given in Table 1, wherefrom it can be observed that the displacement discrepancy at the crack location is now slightly higher $(0.343 \%)$, but still very low (the discrepancy at the same location for the non-cracked case is $0.0245 \%$ ).

The basic polynomial displacement functions produced a value of the first eigenfrequency with a discrepancy of $0.885 \%$, while the upgraded functions yielded even lower discrepancy (-0.099\%). The same basic polynomial displacement functions were also implemented in the buckling load analysis already producing an acceptable discrepancy $(0.991 \%)$. However, the agreement of the results was further improved with the upgrading of the initial polynomial functions when the buckling force error decreased to $0.356 \%$.
Table 1 Comparison of some values for the simply supported beam

\begin{tabular}{lcc}
\hline Parameter & model & value \\
\hline$v_{1}(5 \mathrm{~m})$ & GDEs & $-0.1013 \mathrm{~m}$ \\
$v_{1}(5 \mathrm{~m})$ & 3D FE model & $-0.1009 \mathrm{~m}$ \\
$\omega_{1}$ & basic polynomials & $18.6983 \mathrm{rad} / \mathrm{s}$ \\
$\omega_{1}$ & polynomials upgrade & $18.5159 \mathrm{rad} / \mathrm{s}$ \\
$\omega_{1}$ & 3D FE model & $18.5343 \mathrm{rad} / \mathrm{s}$ \\
$P_{\text {crit }}$ & basic polynomials & $259238 \mathrm{~N}$ \\
$P_{\text {crit }}$ & polynomials upgrade & $257607 \mathrm{~N}$ \\
$P_{\text {crit }}$ & 3D FE model & $256693 \mathrm{~N}$ \\
\hline
\end{tabular}

Table 2 Comparison of some values for the propped cantilever

\begin{tabular}{lcc}
\hline Parameter & model & value \\
\hline$v_{1}(5 \mathrm{~m})$ & GDEs & $-0.04272 \mathrm{~m}$ \\
$v_{1}(5 \mathrm{~m})$ & 3D FE model & $-0.04262 \mathrm{~m}$ \\
$V_{A}$ & GDEs & $12332.3 \mathrm{~N}$ \\
$V_{A}$ & 3D FE model & $12329.7 \mathrm{~N}$ \\
$M_{A}$ & GDEs & $23322.6 \mathrm{Nm}$ \\
$M_{A}$ & 3D FE model & $23290.9 \mathrm{Nm}$ \\
$V_{B}$ & GDEs & $7667.7 \mathrm{~N}$ \\
$V_{B}$ & 3D FE model & $7672.0 \mathrm{~N}$ \\
$\omega_{1}$ & basic polynomials & $29.0211 \mathrm{rad} / \mathrm{s}$ \\
$\omega_{1}$ & polynomials upgrade & $27.9477 \mathrm{rad} / \mathrm{s}$ \\
$\omega_{1}$ & 3D FE model & $27.9544 \mathrm{rad} / \mathrm{s}$ \\
$P_{c r i t}$ & basic polynomials & $588154.4 \mathrm{~N}$ \\
$P_{c r i t}$ & polynomials upgrade & $537350.8 \mathrm{~N}$ \\
$P_{c r i t}$ & 3D FE model & $530869.9 \mathrm{~N}$ \\
\hline
\end{tabular}

\subsection{Propped cantilever}

Transverse displacements along the whole structure obtained from GDE's solutions are presented in Fig. 3 as continuous functions, together with the discrete nodal values from the 3D FE model (discrete dots). The results from both models exhibit excellent agreement, either for the cracked case (in black) as well as for the non-cracked case (in grey).

Table 2 presents some representative example's values. The discrepancy of transverse displacement at the crack location is $0.231 \%$ (and $-0.0299 \%$ for the non-cracked case). Since this beam was statically indeterminate the reactions could not be evaluated from the simple static analysis anymore. However, comparison of the values from the derivatives of transverse displacements functions shows that the discrepancies against the 3D model's values are moderate (up to $0.1360 \%$ ).

The discrepancy $(-0.024 \%)$ was even lower when the value of the first eigenfrequency was obtained from the upgraded polynomials. However, the buckling load analysis produced slightly higher discrepancy $(-1.221 \%)$. 


\subsection{Clamped-clamped beam}

Also for this type where the displacement functions' coefficients have the more complex form the results from both models exhibit overall first-rate agreement, Fig. 4. This is valid for the cracked case (in black) and also for the noncracked case (in grey).

Some representative example's values are summarized in Table 3. The discrepancy of transverse displacement at the crack location is again very low $(0.1704 \%)$. Very small discrepancies were also observed for the evaluated reactions which could not be evaluated from the simple static analysis. The maximal discrepancy when comparing the values from the derivatives of transverse displacements functions against the 3D model's values appeared for the bending moment at the left support $(0.1154 \%)$, while the discrepancies for the other reactions were considerably smaller.

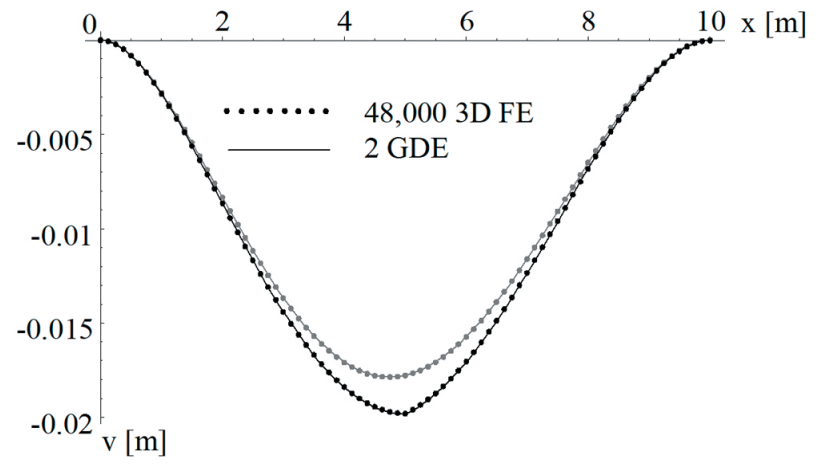

Fig. 4 Comparison of transverse displacements from both applied models

Table 3 Comparisons of some values for the clamped-clampedbeam

\begin{tabular}{lcc}
\hline Parameter & model & value \\
\hline$v_{1}(5 \mathrm{~m})$ & GDEs & $-0.01985 \mathrm{~m}$ \\
$v_{1}(5 \mathrm{~m})$ & 3D FE model & $-0.01981 \mathrm{~m}$ \\
$V_{A}$ & GDEs & $9508.8 \mathrm{~N}$ \\
$V_{A}$ & 3D FE model & $9506.8 \mathrm{~N}$ \\
$M_{A}$ & GDEs & $14709.5 \mathrm{Nm}$ \\
$M_{A}$ & 3D FE model & $14692.6 \mathrm{Nm}$ \\
$V_{B}$ & GDEs & $10491.2 \mathrm{~N}$ \\
$V_{B}$ & 3D FE model & $10492.9 \mathrm{Nm}$ \\
$M_{B}$ & GDEs & $19621.2 \mathrm{Nm}$ \\
$M_{B}$ & 3D FE model & $19610.9 \mathrm{Nm}$ \\
$\omega_{1}$ & basic polynomials & $43.5453 \mathrm{rad} / \mathrm{s}$ \\
$\omega_{1}$ & polynomials upgrade & $42.5610 \mathrm{rad} / \mathrm{s}$ \\
$\omega_{1}$ & 3D FE model & $42.5604 \mathrm{rad} / \mathrm{s}$ \\
$P_{c r i t}$ & basic polynomials & $1082479 \mathrm{~N}$ \\
$P_{c r i t}$ & polynomials upgrade & $1030595 \mathrm{~N}$ \\
$P_{c r i t}$ & 3D FE model & $1044774 \mathrm{~N}$ \\
\hline & & \\
\hline
\end{tabular}

The value of the first eigenfrequency was evaluated almost without any discrepancy $(0.0015 \%)$.

On the other hand, the discrepancy was evidently higher $(-1.357 \%)$ when the buckling load analysis was completed. Nevertheless, the discrepancy evidently decreased $(0.710 \%)$ when basic polynomial functions were replaced by dedicated polynomial functions.

\section{Conclusions}

Simplified computational model of cracked beams was implemented for evaluating transverse displacements and inner forces due to the static bending, first eigenfrequency as well as buckling load for several transversely-cracked slender beams with a linear variation of width. Transverse displacements' functions due to transverse load were firstly evaluated from solutions of coupled differential equations, whilst the implementation of well-known relationships from the Euler-Bernoulli beam theory further allowed for the bending-moments' and shear-forces' functions to be computed.

The results obtained with the discussed approach were afterwards compared to the results obtained from the pure numerical approach implementing 3D finite elements within the framework of the finite element method. Despite the clear differences in the mathematical form and computational efforts between both approaches considered, the considered examples showed that the application of the simplified model produces adequately matching results as no major differences are noticeable in GDEs transverse displacements solutions against 3D FE solutions. When considering the transverse displacements the maximum discrepancy of $0.343 \%$ from all considered examples appeared for the simply supported beam which represents excellent matching of the results. However, it should be noted that the matching of the results is greatly influenced by the value of rotational spring stiffness, for which many definitions given by many authors are available. Furthermore, even the same definition can be differently successful in providing a good agreement for different crack's depth and/or element's boundary conditions.

However, although bending displacements functions could be implemented in first eigenfrequency analysis, basic polynomial solutions were used instead. These functions were afterwards upgraded, providing good matching of the results. This indirectly associates that by applying appropriate transverse displacement functions even higher eigenfrequencies could be evaluated. It can be thus concluded that the model is suitable also for free vibration analyses with non-breathing crack. 
Basic polynomial solutions and their upgraded versions were used also in buckling load analyses, providing good results from the engineering point of view.

Nevertheless, it is reasonable to expect that by implementing more dedicated computational methods for eigenfrequency analysis as well as for buckling analysis this would also reflect in better results from the simplified model.

The Okamura's computational model has thus proved itself to be usable for beams with linear variations of widths for various types of analyses. Furthermore, the transverse bending displacement solutions obtained from differential equations are given in analytical form

\section{References}

[1] Craciun, E.-M., Soós, E. "Anti-plane States in an Anisotropic Elastic Body Containing an Elliptical Hole", Mathematics and Mechanics of Solids, 11(5), pp. 459-466, 2006. https://doi.org/10.1177/1081286505044138

[2] Okamura, H., Liu, H. W., Chu, C.-S., Liebowitz, H. "A cracked column under compression", Engineering Fracture Mechanics, 1(3), pp. 547-564, 1969.

https://doi.org/10.1016/0013-7944(69)90011-3

[3] Dimarogonas, A. D., Papadopulus, C. A. "Vibration of cracked shafts in bending", Journal of Sound and Vibration, 91(4), pp. 583593, 1983.

https://doi.org/10.1016/0022-460X(83)90834-9

[4] Rajab, M. D., Al-Sabeeh, A. "Vibrational characteristics of cracked shafts", Journal of Sound and Vibration, 147(3), pp. 465-473, 1991. https://doi.org/10.1016/0022-460X(91)90494-5

[5] Krawczuk, M., Ostachowicz, W. M. "Influence of a Crack on the Dynamic Stability of a Column", Journal of Sound and Vibration, 167(3), pp. 541-555, 1993.

https://doi.org/10.1006/jsvi.1993.1352

[6] Sundermeyer, J. N., Weaver, R. L. "On crack identification and characterization in a beam by non-linear vibration analysis", Journal of Sound and Vibration, 183(5), pp. 857-871, 1995.

https://doi.org/10.1006/jsvi.1995.0290

[7] Hasan, W. M. "Crack detection from the variation of the eigenfrequencies of a beam on elastic foundation", Engineering Fracture Mechanics, 52(3), pp. 409-421, 1995.

https://doi.org/10.1016/0013-7944(95)00037-V

[8] Skrinar, M., Pliberšek, T. "New linear spring stiffness definition for displacement analysis of cracked beam elements", Proceedings in Applied Mathematics and Mechanics, 4(1), pp. 654-655, 2004. https://doi.org/10.1002/pamm.200410308

[9] Bakhtiari-Nejad, F., Khorram, A., Rezaeian, M. "Analytical estimation of natural frequencies and mode shapes of a beam having two cracks", International Journal of Mechanical Sciences, 78, pp. 193-202, 2014.

https://doi.org/10.1016/j.ijmecsci.2013.10.007

[10] Labib, A., Kennedy, D., Featherston, C. A. "Crack localisation in frames using natural frequency degradations", Computers \& Structures, 157, pp. 51-59, 2015.

https://doi.org/10.1016/j.compstruc.2015.05.001 for various combinations of boundary conditions, which allows for straightforward further analysis of bending moments and shear forces. The proposed solutions thus yield an adequate, as well as accurate enough approach for the modelling of cracked beam structures in engineering situations, where cracks have to be considered during analysis.

\section{Acknowledgement}

The author acknowledges the partial financial support from the Slovenian Research Agency (research core funding No. P2-0129 (A)).

[11] Khiem, N. T., Tran, H. T. "A procedure for multiple crack identification in beam-like structures from natural vibration mode", Journal of Vibration and Control, 20(9), pp. 1417-1427, 2014. https://doi.org/10.1177/1077546312470478

[12] Cao, M., Radzieński, M., Xu, W., Ostachowicz, W. "Identification of multiple damage in beams based on robust curvature mode shapes", Mechanical Systems and Signal Processing, 46(2), pp. 468-480, 2014.

https://doi.org/10.1016/j.ymssp.2014.01.004

[13] Greco, A., Pau, A. "Detection of a concentrated damage in a parabolic arch by measured static displacements", Structural Engineering and Mechanics, 39(6), pp. 751-765, 2011.

https://doi.org/10.12989/sem.2011.39.6.751

[14] Tharp, T. M. "A finite element for edge-cracked beam columns", International Journal for Numerical Methods in Engineering, 24(10), pp. 1941-1950, 1987.

https://doi.org/10.1002/nme.1620241009

[15] Gounaris, G., Dimarogonas, A. "A finite element of a cracked prismatic beam for structural analysis", Computers \& Structures, 28(3), pp. 309-313, 1988. https://doi.org/10.1016/0045-7949(88)90070-3

[16] Biondi, B., Caddemi, S. "Euler-Bernoulli beams with multiple singularities in the flexural stiffness", European Journal of Mechanics A/Solids, 26(5), pp. 789-809, 2007.

https://doi.org/10.1016/j.euromechsol.2006.12.005

[17] Palmeri, A., Cicirello, A. "Physically-based Dirac's delta functions in the static analysis of multi-cracked Euler-Bernoulli and Timoshenko beams", International Journal of Solids and Structures, 48(14-15), pp. 2184-2195, 2011.

https://doi.org/10.1016/j.ijsolstr.2011.03.024

[18] Skrinar, M. "Elastic beam finite element with an arbitrary number of transverse cracks", Finite Elements in Analysis and Design, 45(3), pp. 181-189, 2009. https://doi.org/10.1016/j.finel.2008.09.003

[19] Skrinar, M., Pliberšek, T. "On the derivation of symbolic form of stiffness matrix and load vector of a beam with an arbitrary number of transverse cracks", Computational Material Science, 52(1), pp. 253-260, 2012. https://doi.org/10.1016/j.commatsci.2011.07.013 
[20] Skrinar, M., Imamovic, D. "On the Bending Analysis of MultiCracked Slender Beams with Continuous Height Variations", Periodica Polytechnica Civil Engineering, 62(4), pp. 873-880, 2018. https://doi.org/10.3311/PPci.11897

[21] Skrinar, M. "Computational analysis of multi-stepped beams and beams with linearly-varying heights implementing closed-form finite element formulation for multi-cracked beam elements", International Journal of Solids and Structures, 50(14-15), pp. 2527-2541, 2013. https://doi.org/10.1016/j.ijsolstr.2013.04.005

\section{Appendix A}

$D_{1}=C_{1}$

$D_{2}=C_{2}+\left(C_{3}-D_{3}\right) \cdot L_{1}$

$D_{3}=C_{3}+\frac{2 \cdot C_{4} \cdot E I_{I}}{K_{r}}-\frac{C_{1}}{k \cdot K_{r}}+\frac{E I_{o} \cdot E I_{1} \cdot q}{2 \cdot k^{2} \cdot K_{r}}+\frac{L_{1}{ }^{2} \cdot q}{2 \cdot K_{r}}$

$D_{4}=C_{4}$

\section{Appendix B}

$C_{1}=0$

$C_{2}=\frac{E I_{o}{ }^{2} \cdot L \cdot q}{2 \cdot k^{3}}-\frac{E I_{o} \cdot L^{2} \cdot q}{4 \cdot k^{2}}+\frac{L^{3} \cdot q}{6 \cdot k}+\frac{L_{1}{ }^{3} \cdot q}{2 \cdot K_{r}}$

$-\frac{E I_{o}{ }^{3} \cdot q \cdot \operatorname{Ln}\left(E I_{L}\right)}{2 \cdot k^{4}}$

$C_{3}=\frac{E I_{o} \cdot L \cdot q}{2 \cdot k^{2}}-\frac{L^{2} \cdot q}{4 \cdot k}-\frac{L_{l}{ }^{2} \cdot q}{2 \cdot K_{r}}-\frac{E I_{o}{ }^{2} \cdot q}{2 \cdot k^{3}} \cdot \operatorname{Ln}\left(E I_{L}\right)$

$C_{4}=-\frac{E I_{o} \cdot q}{4 \cdot k^{2}}$

\section{Appendix C}

$C_{1}=\frac{E I_{o}{ }^{2} \cdot q}{2 \cdot k}-\frac{E I_{o} \cdot E I_{L} \cdot q}{2 \cdot k}$

$C_{2}=-\frac{E I_{o}{ }^{2} \cdot E I_{L} \cdot q \cdot \operatorname{Ln}\left(E I_{o}\right)}{2 \cdot k^{2}}$

$C_{3}=\frac{E I_{L} \cdot\left(E I_{o}+E I_{L}\right) \cdot L \cdot q}{4 \cdot k^{2}}+\frac{E I_{o} \cdot\left(E I_{1}+E I_{L}\right) \cdot L_{1} \cdot q}{2 \cdot k^{2} \cdot K_{r} \cdot L}$

$-\frac{L^{2} \cdot q}{12 \cdot k}+\frac{E I_{1} \cdot E I_{L}-E I_{o} \cdot\left(E I_{1}+k \cdot L\right) \cdot q}{2 \cdot k^{2} \cdot K_{r}}$

$-\frac{\left(E I_{o}{ }^{2}+E I_{1} \cdot E I_{L}\right) \cdot L_{1} \cdot q}{2 \cdot k^{2} \cdot K_{r} \cdot L}+\frac{L_{1}{ }^{2} \cdot\left(L_{1}-L\right) \cdot q}{2 \cdot K_{r} \cdot L}$

$+\frac{E I_{o}{ }^{2} \cdot E I_{L} \cdot q \cdot \operatorname{Ln}\left(E I_{o}\right)}{2 \cdot k^{4} \cdot L}-\frac{E I_{o} \cdot E I_{L}{ }^{2} \cdot q \cdot \operatorname{Ln}\left(E I_{L}\right)}{2 \cdot k^{4} \cdot L}$
[22] Pestel, E. C., Leckie, F. A. "Matrix methods in elastomechanics", 1st ed., McGraw-Hill, London, United Kingdom, 1963.

[23] Koloušek, V. "Dynamics in Engineering Structures", 1st ed., Newnes-Butterworth, Kidlington, Oxford, United Kingdom, 1973.

[24] Skrinar, M. "On the application of a simple computational model for slender transversely cracked beams in buckling problems", Computational Materal Science, 39(1), pp. 242-249, 2007. https://doi.org/10.1016/j.commatsci.2006.01.029

$C_{4}=-\frac{E I_{L} \cdot q}{4 \cdot k^{2}}$

\section{Appendix D}

$C_{1}=\frac{E I_{o}{ }^{2} \cdot q}{2 \cdot k}+\frac{C_{2} \cdot k^{3}}{E I_{o} \cdot \operatorname{Ln}\left(E I_{o}\right)}$

$C_{2}=\frac{C_{3} \cdot E I_{o}}{k}$

$C_{3}=\frac{E I_{L} \cdot\left(4 \cdot C_{4} \cdot k+L \cdot q\right) \cdot \operatorname{Ln}\left(E I_{o}\right)}{2 \cdot k^{2}}$

$C_{4}=\frac{q}{12 \cdot k} \cdot\left(-6 \cdot k^{2} \cdot\left(L-L_{1}\right)^{2} \cdot\left(E I_{L}+k \cdot L_{l}\right)+K_{r}\right.$

$\left.\cdot\left(k \cdot L^{2} \cdot\left(6 \cdot E I_{L}+k \cdot L\right)-6 \cdot E I_{L}{ }^{2} \cdot L \cdot \operatorname{Ln}\left(E I_{L} / E I_{o}\right)\right)\right)$

$/\left(-k \cdot K_{r} \cdot L \cdot\left(2 \cdot E I_{L}+k \cdot L\right)+2 \cdot k^{3} \cdot\left(L-L_{1}\right)^{2}\right.$

$\left.+2 \cdot E I_{L}^{2} \cdot K_{r} \cdot \operatorname{Ln}\left(E I_{L} / E I_{o}\right)\right)$

\section{Appendix E}

$C_{3}=\operatorname{Ln}\left(E I_{o}\right) \cdot\left(8 \cdot C_{4} \cdot k^{2} \cdot\left(E I_{1}+K_{r} \cdot L\right)+\right.$

$\left.\left(2 \cdot E I_{o} \cdot E I_{1}-2 \cdot E I_{o}{ }^{2}+k \cdot\left(K_{r} \cdot L^{2}+2 \cdot k \cdot L_{I}{ }^{2}\right)\right) \cdot q\right)$

$/\left(4 \cdot k^{2} \cdot\left(k+K_{r} \cdot \operatorname{Ln}\left(E I_{L} / E I_{o}\right)\right)\right)$

$C_{4}=\left(k^{2} \cdot L \cdot\left(6 \cdot E I_{1} \cdot L_{1}+k \cdot L \cdot\left(3 \cdot L_{l}-2 \cdot L\right)\right) \cdot q\right.$

$+6 \cdot E I_{l} \cdot\left(E I_{o}{ }^{2}-E I_{o} \cdot E I_{l}-k^{2} \cdot L_{l}{ }^{2}\right) \cdot q \cdot \operatorname{Ln}\left(E I_{L} / E I_{o}\right)$

$+K_{r} \cdot\left(3 \cdot k^{2} \cdot L^{3} \cdot q+k \cdot L^{2} \cdot\left(k \cdot L-3 \cdot E I_{L}\right)\right.$.

$\left.q \cdot \operatorname{Ln}\left(E I_{L} / E I_{o}\right)\right) / \Delta$

with

$$
\begin{gathered}
\Delta=12 \cdot k^{2} \cdot\left(k \cdot L \cdot\left(k \cdot L-2 \cdot\left(K_{r} \cdot L+k \cdot L_{l}+E I_{l}\right)\right)\right. \\
\left.+\left(2 \cdot E I_{I}^{2}+\left(E I_{o}+E I_{L}\right) \cdot K_{r} \cdot L\right) \cdot \operatorname{Ln}\left(E I_{L} / E I_{o}\right)\right)
\end{gathered}
$$

